\title{
Dietary Intake in NCAA Division IA Football Players During the Off- Season
}

\section{KATHRYN M MCCAMMON, JONATHAN M OLIVER, JONATHAN TANGUAY, DAVID WEIR, \& STEPHEN F CROUSE}

Texas A \& M University

\section{ABSTRACT}

Off-season football player interaction with athletic personnel is limited and may result in the athletes' diets not meeting current ACSM guidelines for macronutrient intake or recommendations for key micronutrients, having a negative impact on performance and health. Purpose: To examine the dietary intake of NCAA Division IA football players during the summer off-season to determine if ACSM nutrition guidelines were being met. Methods: Fifty-nine NCAA Division IA football players $(20 \pm 2$ yrs, $186.7 \pm 6.4 \mathrm{~cm}, 102.9 \pm 18.5 \mathrm{~kg}$ ) completed a 24 hour dietary recall administered by a Registered Dietitian. Recalls were obtained during the summer off-season when players were not required to participate in scheduled practice or conditioning workouts. Diets were analyzed for nutrient content using Nutribase 8.0 software. Acceptable intake of micronutrients defined as $>75 \%$ Dietary Reference Intake (DRI). Results: All players met $(22 \%)$ or exceeded $(78 \%)$ the recommendation for $\%$ calories from fat. Carbohydrate intake was not met by 54 players (92\%), while $42(71 \%)$ met or exceeded guidelines for protein intake. Results of selected micronutrient analyses are shown in the table.

\begin{tabular}{|l|l|l|l|l|l|l|l|l|}
\hline Micronutrients & $\begin{array}{l}\text { Vitamin } \\
\text { B1 }\end{array}$ & $\begin{array}{l}\text { Vitamin } \\
\text { B2 }\end{array}$ & $\begin{array}{l}\text { Vitamin } \\
\text { B3 }\end{array}$ & $\begin{array}{l}\text { Vitamin } \\
\text { B5 }\end{array}$ & $\begin{array}{l}\text { Vitamin } \\
\text { B6 }\end{array}$ & $\begin{array}{l}\text { Folate } \\
\text { Vitamin } \\
\text { B12 }\end{array}$ & Biotin \\
\hline $\begin{array}{l}\text { Frequency > 75\% } \\
\text { DRI (n = 59) }\end{array}$ & 45 & 57 & 21 & 31 & 56 & 56 & 8 \\
\hline$\%$ of $\mathbf{n}$ & $76 \%$ & $97 \%$ & $36 \%$ & $53 \%$ & $95 \%$ & $36 \%$ & $95 \%$ & $14 \%$ \\
\hline Micronutrients & Vitamin C & Vitamin D & Vitamin E & Calcium & Magnesium & Iron & Selenium & Zinc \\
\hline $\begin{array}{l}\text { Frequency > 75\% } \\
\text { DRI (n=59) }\end{array}$ & 24 & 15 & 9 & 48 & 7 & 58 & 49 & 45 \\
\hline$\%$ of $\mathbf{n}$ & $41 \%$ & $25 \%$ & $15 \%$ & $81 \%$ & $12 \%$ & $98 \%$ & $83 \%$ & $76 \%$ \\
\hline
\end{tabular}

Conclusion: The majority of football players in the off season met or exceeded current protein and fat guidelines, while not consuming adequate carbohydrates. Most micronutrient intakes did not reach current recommendations suggesting players need to be further educated on the performance-related benefits of appropriately balanced diets.

Partial support for this study was provided by Martek Biosciences Corporation ${ }^{\circledR}$ 